\title{
An Efficient Position based Power Aware Routing Algorithm in Mobile Ad-hoc Networks
}

\author{
Md. Mahbubur Rahman \\ CSE Department, Military Institute of Science and Technology, Dhaka, Bangladesh-1217. \\ E-mail: mahbubcse@yahoo.com \\ Md. Akhtaruzzaman \\ Technology Department, Grameen-Intel Ltd, Dhaka, Bangladesh-1217. \\ E-mail: tushar_md@yahoo.com
}

\begin{abstract}
In this paper we introduced an efficient scheme based on a weighted metric of remaining battery power, speed and distance of nodes for determining routes in wireless Mobile Ad hoc Networks (MANET). For the cases where significant difference in the velocities of the communicating nodes or the battery power of the intermediate nodes is low, traditional schemes fail to establish the communication among nodes with reliable QoS. We proposed a new algorithm that uses weighted combination of metrics of distance, velocity and battery power in selecting the route over earlier MFR (Most Forward within Radius) method. The proposed scheme encompasses the load balancing issues and eventually it increases the network lifetime and network performance. Simulation experiment showed that the proposed algorithm reduces the packet loss than that of existing MFR algorithm. Experimental results also revealed that besides packet loss, the proposed strategy achieves higher throughput $(14.35 \%)$ rate than that of existing MFR. Furthermore, usages of these new metrics ensure the higher mean time to node failure.
\end{abstract}

Index Terms - Mobile Adhoc Network, Position based Routing, Speedy Node, Power Efficient, Weight based Algorithm and Balancing Network.

\section{INTRODUCTION}

A Mobile Ad Hoc Network (MANET) is the only means of communication where there is no other network (battle field or ocean operations) or the established network is completely collapsed due to any natural disaster and rescue teams have no other way of communication. Where communicating entities are highly mobile in nature communication using position based routing protocol is the most suitable methodology [1]. Efficient routing among a set of mobile nodes is one of the most important purposes in Mobile Ad-Hoc networks (MANETs). But all the routing protocols do not consider the other MANET factors like speed and battery remaining power. Thereby, we recommend an algorithm that results a more balanced use of the battery capacity of the nodes in the network. We proposed an efficient routing algorithm over existing position based protocols to obtain the following objectives:

i. To reduce packet loss this increases with the throughput of nodes

ii. To extend the service life of MANET

\section{BACKGROUND}

\section{A. Routing}

Routing is the procedure of selecting paths in a network along which to send network traffic or packet. Routing is performed for several types of networks, including the telephone network, electronic data networks (such as the Internet), and transportation networks. Here we are concerned mainly with routing in electronic data networks using packet switching technology. In packet switching networks, routing directs packet forwarding, the transfer of logically addressed packets from their source toward their final destination through intermediate nodes; naturally hardware devices called routers, bridges, gateways, firewalls, or switches are used in routing [2].

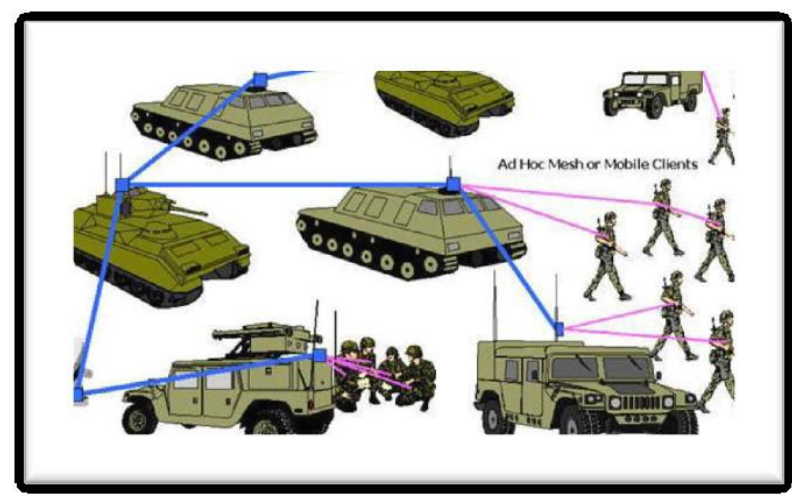

Fig.1. MANET in Military Environment

General-purpose computers with multiple network cards can also advance packets and perform routing, though they are not dedicated hardware and may experience limited performance. The routing process typically directs forwarding on the basis of routing which maintains an algorithm or technique of the routes to reach the network destinations [2]. 


\section{B. Mobile Ad hoc Network (MANET)}

A mobile ad hoc network (MANET), also called a mobile mesh network, is a self-configuring network of mobile devices connected by wireless links. It consists of wireless nodes making a form of a temporary network without the aid of any centralized administration or fixed based-station control. Each mobile node in the network can generate, transmit, receive or route data packets simultaneously. The routing decisions are taken from the neighboring nodes by calculating the nodes distance using GPS (global positioning system) or LBS (Location based Service) and broadcast this location by sending broadcast packets [2].

A preliminary classification of the routing protocols can be done via the type of cast property, whether they use the Unicast, Multicast, Broadcast and Geo-cast forwarding [4].

\section{Routing Protocol types of MANET}

Mobile nodes in an Ad Hoc network join and leave the network totally in an asynchronous manner anytime anywhere. All the existing routing protocols of Ad Hoc networks can be divided into two main categories:

$>$ Topology-based

$>$ Position-based.

Topology-based routing protocols use information about links that exists in the network to perform packet forwarding [6]. Position-based protocols are performed by a scheme based on the updated information and situation of the node.

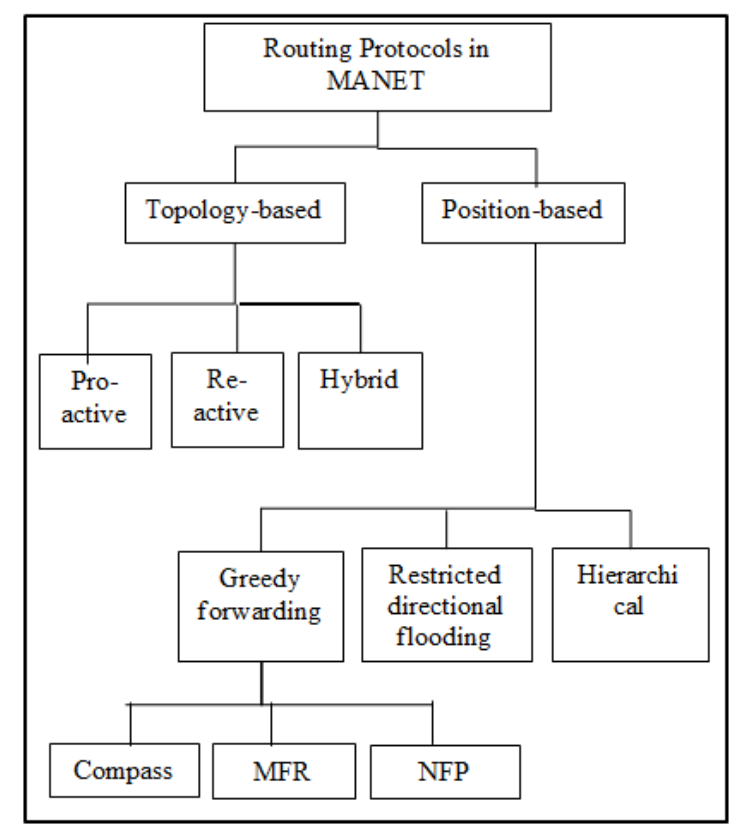

Fig.2. Classification of Routing Protocols in MANET.

Since the beginning of Defense Advanced Research Projects Agency (DARPA) packet radio networks in the early 1970s [4], numerous protocols have been developed for ad hoc mobile networks. Such protocols must deal with the typical limitations of these networks, which include high power consumption, low bandwidth, and high error rates.

In recent developments, position-based routing protocols exhibit better scalability, performance and robustness against frequent topological changes [7]. The primary goal of every routing scheme is to increase the delivery rate as well as reduce the packet loss.

\section{RELATED WORK}

\section{A. Position based routing protocol}

Position based routing protocol uses the geographical position of nodes to make routing decisions, which results in improved efficiency and performance [9].

In the same fashion each intermediate node selects a next hop node until the packet reaches the destination. They periodically broadcast small packets to announce their position and enable other nodes maintain a one-hop neighbor table.

The distance between two neighbor nodes can be estimated on the basis of incoming signal strengths. The destination node is known and addressed by means of its location. Relative coordinates of neighbor nodes can be obtained by exchanging such information between neighbors [3].

\section{B. MFR and DSR Routing Protocol}

MFR [3] or Most Forward within Radius is a common position based routing protocols in which source uses the nearest node within its radius to send the message to the desired destination. In Position based routing, the destination node is determined by means of its location or distance get optimum routes for a particular radio range (see circle in Figure 2 where node 1 and 4 node's range is shown with circle).

The inter-node distance is premeditated by its Euclidean length is given below from the two graphs axis or position [4].

$$
\Delta_{\varepsilon}=\sqrt{\left(x_{2}-x_{1}\right)^{2}+\left(y_{2}-y_{1}\right)^{2}}
$$

But using only the distance metric to find the route where the packet to be sent is not suitable for all conditions [4].

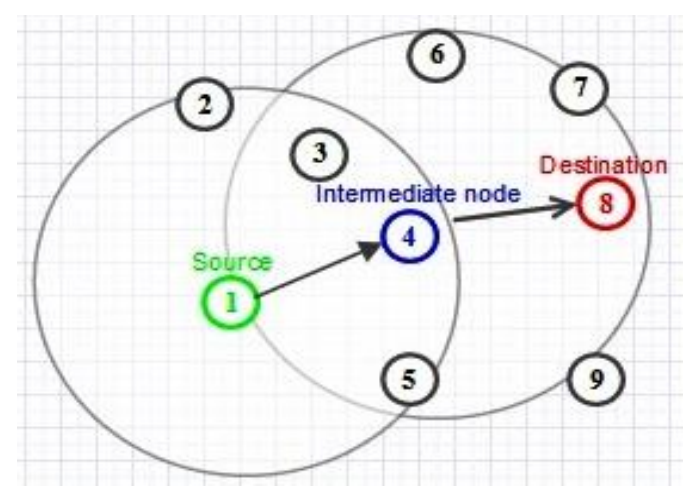

Fig.3. Routing Path from MFR Method. 
In figure 1 , if the source node 1 wants to reach for the destination node 8 , using the traditional MFR method the intermediate node 4 will be selected here.

Dynamic Source Routing (DSR) is one of the more generally accepted reactive routing protocols also known as On-Demand Routing Protocol. In DSR, each neighbor broadcasts this RREQ, adding its own address in the header of the packet. When the RREQ is received by the destination or by a node with a route to the destination, a route reply (RREP) is generated and sent back to the sender along with the addresses accumulated in the RREQ header [6].

\section{Power Concern Routing}

Energy is a source and non-renewable in wireless ad hoc network, energy- efficient protocol design is a key concern. The design and performance analysis of such protocols require proper modeling for the measurement of energy consumption.

The cost associated with each packet at a node is represented as the total of incremental cost " $\mathrm{m}$ " proportional to the packet size and a fixed cost " $b$ " associated with channel acquisition. [12]:

Thus, the cost of a broadcast packet will be of the form

$$
\text { Cost }=\mathrm{m} \times \text { size }+\mathrm{b}
$$

Since mobile nodes are usually battery-operated, one of the major reasons of node failure is battery drain out.

For example, in Figure 2, let's assume that the intermediate node 4 is running with low battery power. If the low battery powered node 4 is chosen in communicating between node 1 and node 8 as intermediate node the battery of node 4 will eventually go down quickly which in turn will impact the network lifetime and QoS.

Each node in the network functions as a mobile router of data packets for other nodes and should maintain the network routes for long standing which is not possible due to limited battery source. Also, due to node mobility, link failures in such networks are very frequent and render certain standard protocols inefficient resulting in wastage of power and loss in throughput. The power consumption is an important issue with the goal to maintain the network lives for long by consuming less power [12].

Lifetime Prediction Routing (LPR) is an on demand source routing protocol that uses battery lifetime prediction. This protocol favors the path whose lifetime is maximum, i.e.

$$
\operatorname{Max}_{\pi} T_{\pi}(t)=\operatorname{Max}_{i \in \pi}\left(T_{i}(t)\right)\left\{\sum_{(i, j) \in \pi} T_{i j}\right\}
$$

Where, $T_{\pi}(t)$ : lifetime of path $\pi$,

$T_{i}(t)$ : predicted lifetime of node $\mathrm{i}$ in path $\pi$ [5].

\section{Problems of MFR and Existing Protocols}

In MFR, nodes focus on distance as deciding factor to forward packet to a neighbor node and it does not consider the node conditions which are not suitable for all circumstances. The main disadvantage of this protocol is it may select the least-power cost routes [8].

If there are situation such as

a) The closest neighbor to target node has very low battery power; it may go down before forwarding the packet which results packet loss.

b) The closest neighbor to target node; may have high or low speed with respect to source or target node speed. When the directions are different, the nodes may speared out and go out of range from the source or target node.

Such critical situations can occur anytime, and then the network link may be down or will face packet loss which decreases network throughput as well as lifetime [9].

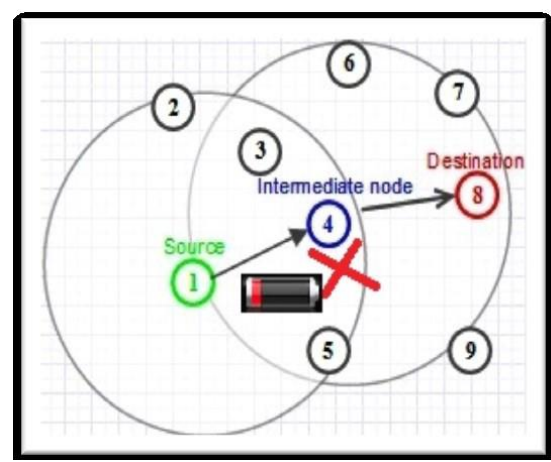

Fig.4. Problems in MFR Method (a node dead situation).

To tackle such situation, when the remaining lifetime decreases and goes beyond a threshold level, the node in a path sends a route error back to the destination as if the route was rendered invalid. The destination sends this route error message to the source. Then the source initiates route discovery again. Node $i$ generate a route error at time $t$ when the following condition is met:

$$
T_{i}(t 0)-T_{i}(t) \leq \delta
$$

Where,

$\delta$ : change threshold for lifetime prediction

t0: route discovery time, $t$ : current time

Ti: predicted lifetime of node $\mathrm{i}$ [5]. But detecting the vulnerable node and then initiating a new route discovery is time consuming.

The hybrid combination of Ant Colony Optimization (ACO) and Multi Agent System (MAS) [13] also cannot manage the above situation for ensuring a balanced MANET

Also Temporarily Ordered Routing Algorithm (TORA) and Associativity Based Routing (ABR) have some challenges to avoid this node dead situation in their comparative study [14].

Therefore, we proposed an efficient position based power-aware routing algorithm in mobile ad-hoc networks which considers the power and velocity issues 
in advance. Thus the proposed scheme addresses the load balancing issues and eventually increases the network lifetime and network performance.

\section{PROPOSED SCHEME}

In our approach, a modified RREQ is sent out and the entry in the route cache corresponding to the node that has moved out of range is purged. We have introduced four new variables to measure the node metric and cost, so the additional information are included in the modified packet format accordingly.

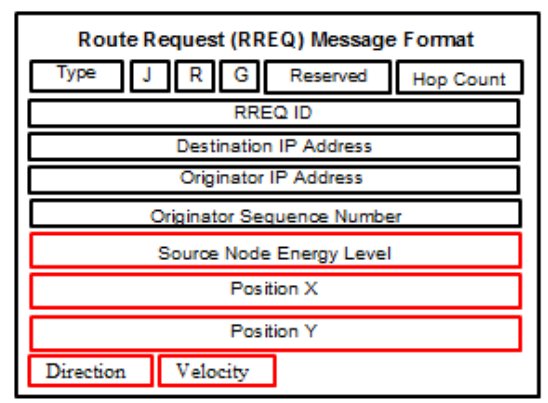

Fig.5. Modified RREQ Message Structure.

Figure-4 shows the modified RREQ packet format through which every node communicates and exchanges their information where they have the necessary protocol information [5].

Our proposed algorithm is described below:

a) Select nodes, $\mathrm{J}=\left\{\mathrm{N}_{\mathrm{i}}\right\}$ where $\mathrm{N}_{\mathrm{i}}$ is within the range of $\mathrm{S}$ toward the direction of target what we get from RREQ message of above like Fig-2.

b) For every $\mathrm{N}_{\mathrm{i}}$, Calculate, Distance, $=\sqrt{\left(x_{2}-x_{1}\right)^{2}+\left(y_{2}-y_{1}\right)^{2}} / / D_{i}=$ distance between two nodes: 1 (Source) and 2(intermediate).

c) Calculate, Relative speed, $R_{i}=\left|S_{S}-S_{i}\right|$; // $S_{S}$ and $S_{i}$ are the speeds of Source node $S$ and intermediate node $\mathrm{N}_{\mathrm{i}}$

d) Compute, Cost $\mathrm{Z}_{\mathrm{i}}=\left(\mathrm{D}_{\mathrm{i}} * \mathrm{~W}_{\mathrm{d}}\right)-\left(\mathrm{P}_{\mathrm{i}} * \mathrm{~W}_{\mathrm{p}}\right)+\left(\mathrm{R}_{\mathrm{i}} * \mathrm{~W}_{\mathrm{r}}\right)$; //Where $\mathrm{P}_{\mathrm{i}}, \mathrm{D}_{\mathrm{i}}$ and $R_{i}$ are remaining power, distance and relative speeds respectively.

//here, $W_{p}, W_{d}$ and $W_{r}$ are the weight of remaining power, distance and relative speeds.

e) Select, $\operatorname{MIN}\left\{Z_{i}\right\}$ from every $\mathrm{N}_{\mathrm{i}}$;

f) Transmit data through $\operatorname{MIN}\left\{Z_{i}\right\}$;

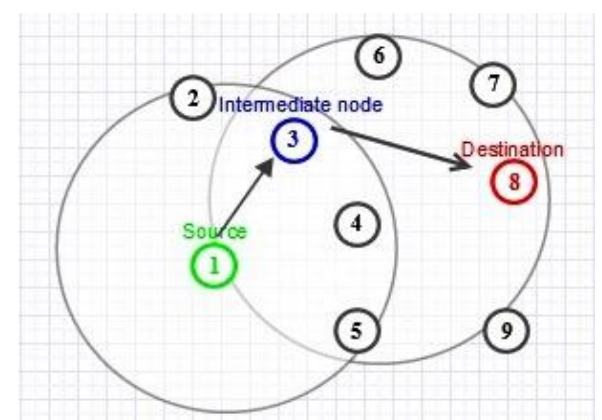

Fig.6. Path Selection through Simulation of Proposed Scheme.
Where we can control the preference by changing the weights $W_{p}, W_{d}$ and $W_{r}$ any time.

Relative speed towards direction will carry a negative value and opposite will carry a positive value. In this proposed model, the preference order for selecting weights is followed in accepted ways [10]:

$\begin{array}{ll}> & \text { Distance } \\ > & \text { Battery Remaining Power } \\ > & \text { Relative Speed. }\end{array}$

Table 1. Sample Calculation of Total Cost from Figure-3 According to the Proposed Algorithm.

\begin{tabular}{|c|c|c|c|c|}
\hline \multirow{2}{*}{$\begin{array}{c}\text { Selected Nodes } \\
\text { position, }\end{array}$} & \multicolumn{4}{|c|}{ Weight Calculation From Proposed } \\
$\mathbf{N}_{\mathbf{i}}$ & $\begin{array}{c}\text { Algorthm } \\
\text { Relative } \\
\text { Distance in } \\
\text { meter, } \boldsymbol{D}_{\boldsymbol{i}}\end{array}$ & $\begin{array}{c}\text { Battery } \\
\text { Power } \\
\text { in \%, } \boldsymbol{P}_{\boldsymbol{i}}\end{array}$ & $\begin{array}{c}\text { Relative } \\
\text { Speed in } \\
\text { meter/ } \\
\text { second, } \boldsymbol{S}_{\boldsymbol{i}}\end{array}$ & $\begin{array}{c}\text { Cost, } \\
\boldsymbol{Z}_{\boldsymbol{i}}\end{array}$ \\
\hline$(120,130) \mathrm{N}_{3}$ & 86.02 & 70 & 5.5 & 21.52 \\
\hline$(140,80) \mathrm{N}_{4}$ & 72.80 & 30 & 6.5 & 49.30 \\
\hline$(90,20) \mathrm{N}_{5}$ & 44.72 & 15 & 7.2 & 36.92 \\
\hline$(160,10) \mathrm{N}_{6}$ & 102.95 & 55 & 4.8 & 52.75 \\
\hline
\end{tabular}

A sample calculation is shown in Table 1 for the topology shown in Figure 3. Here, it is seen that the minimum cost will be incurred if node 3 is chosen. So, according to the proposed method, the routing path will be: $1-->3--->8$.

Here we can see that the node 3 has higher battery power than the other two comparable nodes, adjustable speed with respect to the source and destination node, also its range or distance is not out of coverage. As a result, the proposed algorithm will select node 3 rather than the node 4 which would be chosen by existing MFR method.

Though node 2 may have the highest battery power than node 3 but, this method avoids node 2 as an intermediate node. Other power-aware routing algorithm will choose node 2 in that case. The reason of not choosing node is in fact, it may have relative speed which eventually sends the node out of the range and this is not most forwarded to the destination path.

In summary, the proposed scheme uses priority based combination of metrics of battery power, velocity and distance in selecting the best node through which packet will be delivered. To prolong the lifetime of MANETs, we have to maximize the battery back-up of a mobile node at the same time selecting the mobile nodes which will remain within the coverage [12]. We also proposed to balanced use of nodes battery power in an efficient way over MFR and DSR routing.

\section{EXPERIMENTAL RESULT ANALYSIS}

\section{A. Simulations}

The proposed method has been implemented using Network Simulator (NS-2.35). We designed Existing and 
proposed strategy, with network topology generator software named NAM 1.1.

Table 2. Simulation Parameters

\begin{tabular}{|l|l|l|}
\hline Parameters & Greedy MFR & Proposed Algorithm \\
\hline MAC protocol & 802.11 & 802.11 \\
\hline Number of nodes & 9 & 9 \\
\hline Mobility model & Random way point & Random way point \\
\hline Transmission range & $500 \mathrm{~m}$ & $500 \mathrm{~m}$ \\
\hline Terrain range & $1000 \mathrm{~m} * 1000 \mathrm{~m}$ & $1000 \mathrm{~m} * 1000 \mathrm{~m}$ \\
\hline PHY layer & Phy Wireless & Phy Wireless \\
\hline Queue type & DropTail/PreQ & DropTail/PreQ \\
\hline Simulation & $300 \mathrm{~s}$ & $300 \mathrm{~s}$ \\
\hline
\end{tabular}

There were nine (9) mobile nodes, sender and destination nodes, and others were intermediate nodes. We executed this simulation for the 5 simulations in the same environment by varying their weights $( \pm 2, \pm 3) W_{p}$, $W_{d}$ and $W_{r}$ and their outputs received and analyzed with NS-2 Wireless Trace Analyzer.

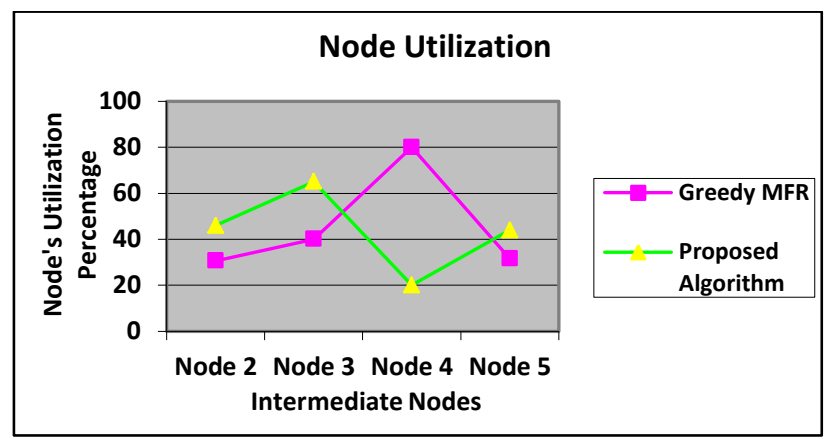

Fig.7. Node Utilization Graph after 300 Second Simulation on Certain Situation.

In the Figure 6, it is depicting that In Greedy MFR had used node 4 maximum times (approximately 80\%) until it goes dead. But in contrast, in spite of using node 4 in our algorithm it used node 3 and did used node 4 around $20 \%$ because it had a lower battery power.

\section{B. Packets Delivery Rate (PDR)}

The primary goal of every routing scheme is to increase the delivery rate as well as reduce the packet loss [11].

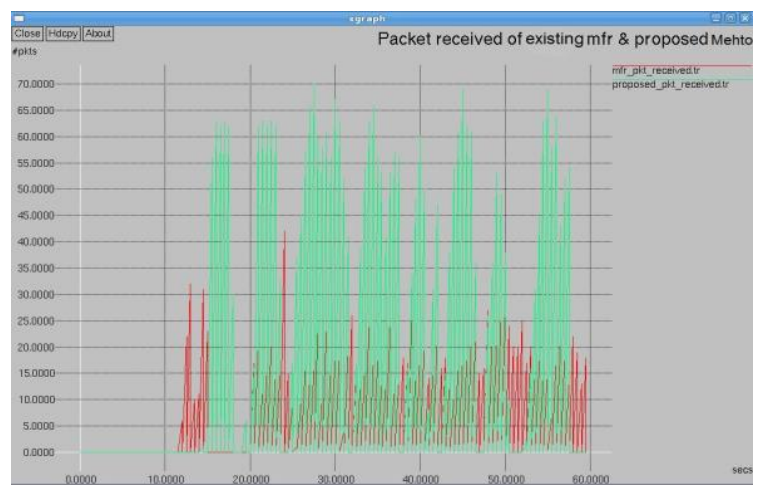

Fig.8. Packet Delivery Comparison Graph in 60 Second.

PDR is calculated in percent and it is expressed by the following formula [15]:

$$
\mathrm{PDR}=\frac{\sum \text { Recieved packets }}{\sum \text { Send packets }} \times 100
$$

The following table .3 shows the throughput or PDR.

Table 3. Minimum, Maximum and Average Throughput of Existing MFR and Proposed Scheme.

\begin{tabular}{|c|c|c|c|}
\hline \multirow{2}{*}{$\begin{array}{c}\text { Throughput of } \\
\text { Schemes }\end{array}$} & \multicolumn{3}{|c|}{$\begin{array}{c}\text { Throughput Comparison between Existing } \\
\text { MFR and Proposed Algorithm. }\end{array}$} \\
\cline { 2 - 4 } & Lowest $^{b}$ & Highest $^{b}$ & Average \\
\hline Existing MFR & 0.14 & 536 & $78.01 \%$ \\
\hline $\begin{array}{c}\text { Proposed } \\
\text { Scheme }\end{array}$ & 0.01 & 752 & $92.36 \%$ \\
\hline
\end{tabular}

From the above table we can see that the proposed method yield better throughput than that of the existing MFR scheme.

\section{Packet Loss Rate}

The proposed method successfully reduced packet loss than that of existing MFR scheme which can be seen from the summary shown in the table below. The packet loss rate was compared with existing MFR.

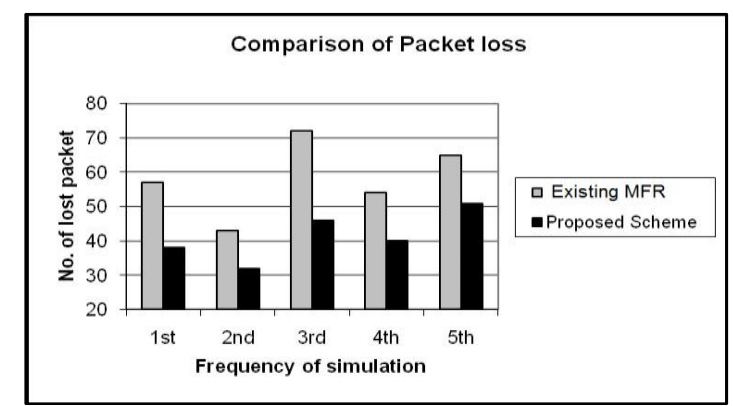

Fig.9. Packet Loss Comparison after 300 Second Simulation on Certain Situation.

From the experimental result shown in figure 6 we can see that the overall packet received has been increased in the proposed scheme.

Table 4. Packets Loss Comparison from the Sample Network.

\begin{tabular}{|c|c|c|c|}
\hline \multirow[b]{2}{*}{ Simulations } & \multicolumn{3}{|c|}{ Packets Loss Calculation } \\
\hline & $\begin{array}{l}\text { Packets loss in } \\
\text { actual in total }^{a}\end{array}$ & $\begin{array}{c}\text { Average of } 5 \\
\text { sample }\end{array}$ & $\begin{array}{c}\text { In } \\
\text { percentage }\end{array}$ \\
\hline Existing MFR & 291 & 58.2 & $21.99 \%$ \\
\hline $\begin{array}{l}\text { Proposed } \\
\text { Scheme }\end{array}$ & 207 & 41.4 & $7.64 \%$ \\
\hline
\end{tabular}

\section{Remaining Energy of Nodes (REN)}

In our simulated network there are 9 nodes that their coordinates is determined randomly. Our testing for the remaining energy of network nodes comprises of selecting 1-4-8 nodes from Greedy MFR method and 1-38 nodes from our proposed method. The variance of remaining energy of nodes had been minimized, especially with our proposed protocols, which finally leads to the stability of the network; see Figure 6. 


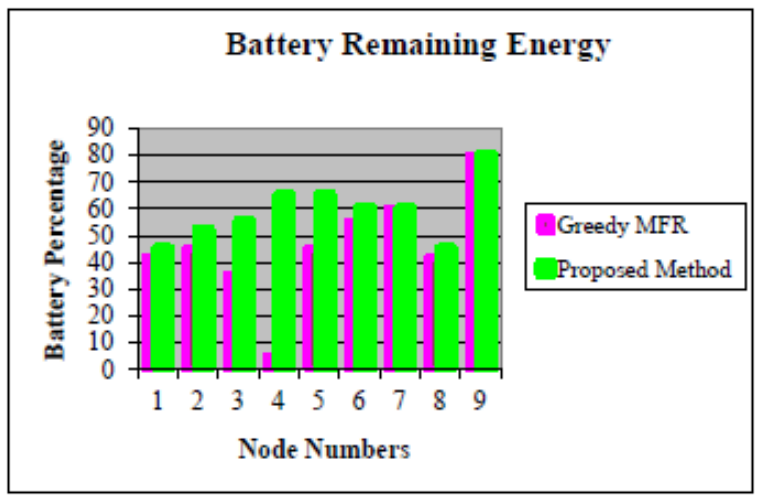

Fig.10. Remaining Battery Power Comparison after 300 Second Simulation on Certain Situation.

\section{E. Delay Average of End-To-End Network}

The average amount of time that one packet takes from source to destination, is specified as the delay packet. End-to-End delay of packets is characterized by these parameters: propagation delay, queuing delay, transmission delay and processing delay [15].

$$
\text { Average End To End Delay }=\frac{\sum \text { Arraive Time }-\sum \text { Sent Time }}{\sum \text { Send packets }}
$$

Table 5. Packet Delay for Greedy MFR and Proposed Scheme

\begin{tabular}{|c|c|c|c|}
\hline Scheme & Minimum & Maximum & Average \\
\hline $\begin{array}{c}\text { Greedy } \\
\text { MFR }\end{array}$ & $\begin{array}{c}0.000936802 \\
(\mathrm{sec})\end{array}$ & $\begin{array}{c}0.93698968 \\
(\mathrm{sec})\end{array}$ & $\begin{array}{c}0.083337618 \\
(\mathrm{sec})\end{array}$ \\
\hline $\begin{array}{c}\text { Proposed } \\
\text { Scheme }\end{array}$ & $\begin{array}{c}0.000840719 \\
(\mathrm{sec})\end{array}$ & $\begin{array}{c}0.18695047 \\
(\mathrm{sec})\end{array}$ & $\begin{array}{c}0.012050933 \\
(\mathrm{sec})\end{array}$ \\
\hline
\end{tabular}

In these types of dynamic environments, the proposed algorithm considers all the parameters (3 parameters of all nodes) to find out a better route for the MANET to make the system to survive as much as possible.

It is a hybrid algorithm of position based and poweraware schemes with parameter tuning options by the operators or network administrator. Here we have avoided the node failure situation due to power exhaustion and skipped the risky nodes as router to make the network a healthy one.

\section{CONCLUSION}

In this paper, we proposed an efficient position based MFR routing scheme with its qualitative evaluation. The method is scalable and resilient to topology changes. We presented a new weight based metric which encompasses the distance deciding metric, remaining battery power and relative speed in selecting next node to deliver packets compared to distance deciding metric used in traditional Existing MFR routing algorithms. The main goal of the proposed scheme is to find- out most suitable intermediate nodes with minimum packet loss and maximum network life time. The proposed algorithm considers the power and velocity issues in advance in selecting intermediate nodes addressing the load balancing issues and eventually increasing the network lifetime and network performance. Besides low packet loss, experimental results also reveal that the proposed strategy achieves higher throughput $(14.35 \%)$ rate than that of existing MFR.

\section{REFERENCES}

[1] S Kaur and A K. Gupta, "Position Based Routing in Mobile Ad-Hoc Networks: An Overview", 1RIMT IET, Punjab, India 2 Dept. of CSE, RIMT IET, Punjab, India, IJCST Vol. 3, Iss ue 4, Oct - Dec 2012.

[2] H. Ashtiani, S. Alirezaee, S. M. M. Hosseini and H. Khosravi, "PNR - New Position based Routing Algorithm for Mobile Ad Hoc Networks", In: Proceedings of the World Congress on Engineering, 2009, Vol I, WCE 2009, July 1 - 3, 2009.

[3] M. Abdoos, K. Faez and M. Sabaei, "Position Based Routing Protocol with More Reliability in Mobile Ad Hoc Network", World Academy of Science, Engineering and Technology, Vol. 3, No 1, pp.304-307 2009.

[4] L. K. Qabajeh, L. M. Kiah and M. M. Qabajeh, "A Qualitative Comparison of Position-Based Routing Protocols for Ad-Hoc Networks", IJCSNS-International Journal of Computer Science and Network Security, Vol. 9 No.2, pp. 131-140, February, 2009.

[5] M Maleki, K Dantu and M Pedram, "Lifetime Prediction Routing in Mobile Ad Hoc Networks" Wireless Communications and Networking, WCNC 2003. IEEE (Volume: 2), 2003.

[6] C. Gandhi and V. Arya, "A Survey of Energy-Aware Routing Protocols and Mechanisms for Mobile Ad Hoc Networks", Proceedings of the International Conference on Advanced Computing, Networking, and Informatics, India, (ICACNI 2013), pp. 111-117, June 14-16, 2013.

[7] S. H. Kang, G. Jeon and Y. Lee, "Improved Energy Aware Routing Protocol in Mobile Ad Hoc Network", Convergence and Hybrid Information Technology, Lecture Notes in Computer Science (LNCS) Vol. 7425, pp. 106-113, 2012.

[8] C. Gu, Q. Zhu, "An Energy-Aware Routing Protocol for Mobile Ad Hoc Networks Based on Route Energy Comprehensive Index", Wireless Personal Communications, Vol. 79, Issue 2, pp. 1557-1570, November, 2014.

[9] M. Buvana, M. Suganthi and K. Muthumayil, "Effective Load Balancing Method in ad hoc Network using PSO", International Journal of Mobile Network Communications \& Telematics ( IJMNCT) Vol. 4, No.5,October 2014.

[10] S. Srivastava, A.K. Daniel, R. Singh and J.P. Saini, "Energy-Efficient Position Based Routing Protocol for Mobile Ad Hoc Networks", International Conference on Radar, Communication and Computing (ICRCC), pp.1823, 21 - 22, December, 2012.

[11] T Suzuki, A Khan, M Kobayashi and W Takita, "Collaboration in Routing and Velocity Measurement Function to Reduce Battery Consumption for Mobile Ad Hoc Networks", International Journal of Hybrid Information Technology Vol. 1, No. 2, April, 2008.

[12] B.Ruxanayasmin, B.Ananda Krishna, T.Subhashini, "Minimization of Power Consumption in Mobile Ad hoc Networks", I.J.Computer Network and Information Security, 2014, 2, 38-44.

[13] H.Vignesh Ramamoorthy, Dr.D.Suganya Devi, "A New Proposal for Route Finding in Mobile AdHoc Networks", 
I. J. Computer Network and Information Security, 2013, 7, $1-8$.

[14] Elizabeth M. Royer, Chai-Keong Toh, "A Review of Current Routing Protocols for Ad Hoc Mobile Wireless", IEEE personal communication, Vol. 6, Issue 2, PN. 46-55, 1999.

[15] Gozali, K. Borna, "SMAODV: A Novel Smart Protocol for Routing in Ad-hoc Wireless Networks Using the PageRank Algorithm", I. J. Computer Network and Information Security, 2015, 9, 46-53.

\section{Authors' Profiles}

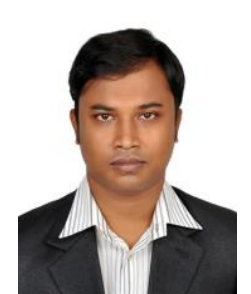

Md. Akhtaruzzaman born at Rajshahi in Bangladesh on 24th October, 1987. He achieved a four years bachelor degree in computer science and engineering from Khulna University in 2010, Bangladesh. His major courses were Operating System, Computer Networks, Artificial Intelligence, Computer Security, Compiler and etc.
Due to his interest in networking he started research and thesis in computer networking and also completed a CCNA course. In 2010 he joined largest IT company of Bangladesh; Grameen Phone IT as a System Engineer. Now, he is working in Grameen-Intel Social Business Limited as Senior Engineer in System and Network Administrator role in Dhaka, Bangladesh. $\mathrm{He}$ expertise job responsibilities include managing and administering Linux, Cisco and other networking devices, Windows Servers, Virtualization and Cloud technologies.

Mr. Akhtaruzzaman is member of IEB, Bangladesh and has published papers in international conference proceedings.

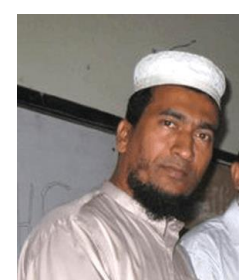

Md. Mahbubur Rahman is a Professor of CSE Department, Military Institute of Science and Technology, Dhaka, Bangladesh. He obtained his $\mathrm{PhD}$, from JAIST, Japan, M.Engg degree from AIT, Thailand and B.Sc.Engg degree from BUET, Bangladesh. He research interests include networks and security, pattern recognition, image processing and data mining.

How to cite this paper: Md. Mahbubur Rahman, Md. Akhtaruzzaman,"An Efficient Position based Power Aware Routing Algorithm in Mobile Ad-hoc Networks", International Journal of Computer Network and Information Security(IJCNIS), Vol.8, No.7, pp.43-49, 2016.DOI: 10.5815/ijcnis.2016.07.06 